\title{
Two methods of weaning persons with quadriplegia from mechanical ventilators
}

\author{
W Peterson MD, W Charlifue MA, A Gerhart RPT MS, G Whiteneck PhD \\ Craig Hospital \& Swedish Medical Center, c/o Craig Hospital Research Department, \\ 3425 South Clarkson, Englewood, Colorado 80110, USA.
}

Two commonly-used methods of weaning individuals with quadriplegia from mechanical ventilators were compared. The medical records were reviewed of 52 patients who were injured at the C3 or C4 levels, were ventilator dependent following their injury, and were treated at the participating facilities. The study subjects had a total of 82 separate wean attempts: 26 utilized intermittent mandatory ventilation, 34 used progressive ventilator-free breathing (T-piece weaning) and 22 used either a combination of these approaches or a different approach. With an overall weaning success rate of $83 \%$, this study demonstrated that individuals with $\mathrm{C} 3$ and $\mathrm{C} 4$ quadriplegia are indeed candidates for ventilator weaning. Comparing the two weaning methods, progressive ventilator-free breathing was found to be successful more often than intermittent mandatory ventilation $(67.6 \%$ compared to $34.6 \%)$. In addition, progressive ventilator-free breathing appeared superior in its ability to provide a more functional alternative to individuals unable to wean completely.

Keywords: respirator weaning; quadriplegia; ventilator; mechanical; weaning; intermittent positive pressure ventilation; IMV.

\section{Introduction}

Over the past several decades the numbers of mechanically ventilated trauma, medical and postsurgical patients who survive have increased dramatically. ${ }^{1}$ With this increased survival, there arose the need to develop specific criteria for and new and varied methods of withdrawing, or 'weaning' these patients from mechanical ventilators. Although criteria have been established which provide some empirical basis for determining when individuals may be ready to wean,$^{2-4}$ there is no apparent consensus on which factors predict successful weaning or on which method of weaning is best. $1.3 .5-9$

Although many different weaning techniques may be employed, two distinct methods of weaning are used commonly. The first, intermittent mandatory ventilation (IMV) is reported to be the method of choice for a wide variety of medical and postsurgical conditions in as many as $90 \%$ of the community hospitals in the United States. ${ }^{10}$ The IMV approach to weaning gradually reduces the ventilator's rate while allowing the patient to take spontaneous breaths in between those respirations that the machine provides. ${ }^{11}$ Some of the reported advantages of IMV include less patient anxiety and discomfort due to its gradual weaning approach; a lower incidence of respiratory alkalosis; a theoretically lower incidence of atelectasis because of the machine's ability to retain a functional residual capacity; 4.5 and the convenience that the IMV approach itself offers. Regarding the latter, IMV weaning requires no additional patient supervision or equipment. It continues with a ventilator system that is already familiar to the patient; the only alteration needed is to the ventilator's respiratory rate..$^{4.9}$ On the other hand, some of the reported disadvantages of IMV are its tendency to prolong the weaning process, ${ }^{5}$ and the contention that IMV offers respiratory musculature neither a prolonged period of conditioning and strengthening, nor a significant, uninterrupted rest period. ${ }^{4.5 .9}$ 
The other method of weaning is termed T-piece weaning, or progressive ventilatorfree breathing (PVFB). It is based on slowly increasing the periods of time that the ventilator dependent individual spends breathing independently of the machine. Its advantage is that it alternates periods of work with periods of rest, thus, in theory, offering respiratory musculature the benefits of a training effect - both for strengthening and endurance. ${ }^{4.9}$ Another advantage of PVFB is the lower airway resistance of the T-piece system. ${ }^{+}$Most of its possible disadvantages seem to result from the abrupt physiological transitions required when going on and off the ventilator repeatedly. These may result in a sudden increase in venous return, a temporary decrease in functional residual capacity due to the loss of the positive-pressure ventilation, and overall stress to the respiratory reserves. ${ }^{4.5}$

Fortunately, for the vast majority of medical patients-particularly those recovering from surgery and anesthesia-return of respiratory function and extubation occur quite quickly; how they were weaned from the mechanical ventilator probably does not matter. ${ }^{4.12}$ It is for the more involved patient - the individual who has had 'prolonged ventilation', who has been ventilator dependent for a week or more, or who has failed at previous weaning attempts - that weaning becomes a more complex issue. ${ }^{3}$ For these patients, the few established weaning predictors and criteria that do exist become less helpful the longer mechanical ventilation has continued..$^{3.5}$

Individuals with quadriplegia meet all of the difficult-to-wean criteria. Frequently, the cause of their respiratory difficultiesneurologically compromised respiratory musculature-remains unresolved. Many will have been dependent upon mechanical ventilators for prolonged periods before weaning is even considered and, within this population, additional pulmonary complications abound. One author estimates that overall $68 \%$ of spinal cord injury survivors are at risk for respiratory complications such as atelectasis and pneumonia. ${ }^{13}$ For those who do experience such complications, the mortality rate is high and death from respiratory complications accounts for half of all deaths during the initial acute hospitalization. ${ }^{14}$ Nonetheless, it has been reported that the majority of those with quadriplegia do eventually wean, ${ }^{15}$ although the time period can be quite protracted. One author reports that it can take as long as 14 months; ${ }^{16}$ another from 2 to 8 years. ${ }^{17}$

In light of the cost, time, and quality of life implications for both patients with quadriplegia and their families, any advantage of one weaning approach over another needs to be identified and documented. Consequently, this investigation was undertaken with the objective of evaluating the relative effectiveness of two methods of weaning individuals with quadriplegia from mechanical ventilators: intermittent mandatory ventilation, and progressive ventilator-free breathing.

\section{Methodology}

The sample of this retrospective study was comprised of individuals admitted for the first time to one of the components of the Rocky Mountain Regional Spinal Injury System in Englewood, Colorado. These include Craig Hospital's spinal cord injury rehabilitation program, and Swedish Medical Center's neurotrauma unit. Specific inclusion criteria required that:

1 the patient's hospital admission was part of his or her initial postspinal cord injury acute care and rehabilitation;

2 the patient was on a mechanical ventilator at the time of admission, or had been successfully weaned from a ventilator at a referring facility;

3 the neurological injury was at the $\mathrm{C} 3$ or $\mathrm{C} 4$ functional level, defined as the presence of functional trapezius muscles (grade 3, 4, or 5), and nonfunctional distal musculature (grade 2 or less, including deltoids and biceps). The first postinjury manual muscle test, performed and documented by the staff physical and occupational therapists, served as the source for this information. These particular levels of injury were selected because ventilator-free breathing is theoretically possible at the C3 and C4 levels, ${ }^{13.15}$ but the weaning process tends to be extremely difficult. Individuals with 
neurologically complete spinal cord injuries above C3 are not likely to wean successfully, while the majority of those with injuries below $\mathrm{C} 4$ either need no ventilator support at all, or wean quickly and easily.

For this study, the start of a weaning attempt was defined as utilizing IMV at a rate less than ten breaths/minute, or PVFB for a minimum of 1 minute. A weaning attempt was considered to be successful when full-time removal from the ventilator for 2 weeks or more had been achieved. A failure was considered to have occurred if the wean attempt was abandoned and not restarted within 14 days. Finally, a third possible outcome-discharge-was used to classify both individuals who were involved in a wean attempt that was not continued after transfer to another hospital, and those who were discharged home but were only partially weaned from the ventilator. Because of the retrospective nature of this study, which relied on review of medical records information obtained from many referring institutions, weaning readiness criteria were those that were in use at the referring hospitals, and thus could not be controlled for. Although it is recognized that ventilators differ with respect to air flow resistance ${ }^{18}-a$ factor that may impact weaning, particularly for the patient using the IMV method - this information was also similarly unavailable to the investigators.

Fifty-two patients admitted consecutively between 1983 and 1990 met all of the criteria for the study. For these 52 patients, complete medical records were obtained from all of the hospitals that had treated them since their spinal cord injuries, including the two facilities where the investigation was conducted. Comprehensive review of the records included an examination of the physicians' orders, consultants' reports, the notes of attending physicians, nurses, and respiratory therapists, ventilator flow sheets, wean sheets, blood gases, and other laboratory and $\mathrm{x}$-ray reports.

Among the 52 persons in the study group, there were 82 separate weaning attempts. Thirty-five of these attempts took place at either Craig or Swedish Hospital, and one more was continued without interruption upon arriving at Craig Hospital; they resulted in 25 successful weans. The remaining 46 attempts occurred at hospitals prior to the patient's transfer to one of the investigating facilities. These 46 attempts led to 18 successful weans.

Twenty-six of the 82 wean attempts utilized the IMV methodology, 34 utilized PVFB, and 22 employed an array of widely varied techniques, including a combination of the two approaches, or a completely different approach, such as continuous positive airway pressure (CPAP), pressure support, or immediate extubation. For this report, the results for the IMV and the PVFB groups were analyzed in detail and are the focus of the ensuing discussion.

It is important to note that there were important differences between the two study groups. Specifically, there was considerable confounding by method of wean and by where the wean was performed. Nearly all of the IMV attempts were conducted relatively earlier, at hospitals other than the two investigating facilities, while nearly all of the PVFB attempts were made later, at Craig and Swedish hospitals. However, an attempt to minimize these confounding factors was made during data analysis by comparing each method's success based upon whether it was used early postinjury (ie during the first month), or greater than 1 month following onset. Data analysis included a variety of cross-tabulations with chi square and Fisher's exact tests for significance.

\section{Results}

The success rate for PVFB was significantly higher: $67.6 \%$ compared with $34.6 \%$ for IMV $(p=0.02)$. Both of these methods resulted in an essentially equal number of discharges without complete success: $19.2 \%$ of IMV attempts versus $17.6 \%$ of PVFB attempts (Table I).

Overall, the eventual outcomes for the 52 patients in the study included two deaths (one by patient choice, one as a result of pulmonary embolus), one discharge to home with full-time ventilator use, six dis- 
charges with part-time ventilator dependence (ie partially weaned), and 43 successful weans. The latter included nine of the 26 IMV attempts, 23 of the 34 PVFB attempts, and 11 of 22 attempts using other methods. The weaning attempt results for the IMV and PVFB subjects are illustrated in Table I. Compared with IMV, the odds ratio ${ }^{19}$ for successful weaning using PVFB was 3.94, with a $95 \%$ confidence interval of 1.19 10.38. This seems to indicate the existence of what is virtually a fourfold likelihood of success with the PVFB method of ventilator weaning.

Figure 1 compares the two weaning

Table I Final wean results (based on number of attempts)

\begin{tabular}{lrrr}
\hline Method & Fail & Success & \multicolumn{1}{c}{$\begin{array}{l}\text { Partial } \\
\text { wean }^{\mathrm{a}}\end{array}$} \\
\hline IMV & $12(46.2 \%)$ & $9(34.6 \%)$ & $5(19.2 \%)$ \\
PVFB & $5(14.7 \%)$ & $23(67.6 \%)$ & $6(17.6 \%)$ \\
\hline
\end{tabular}

aPartial wean $=$ IMV wean in progress at the time of transfer to another hospital, or discharge to home with patient ventilator free part of the day, utilizing T-piece.

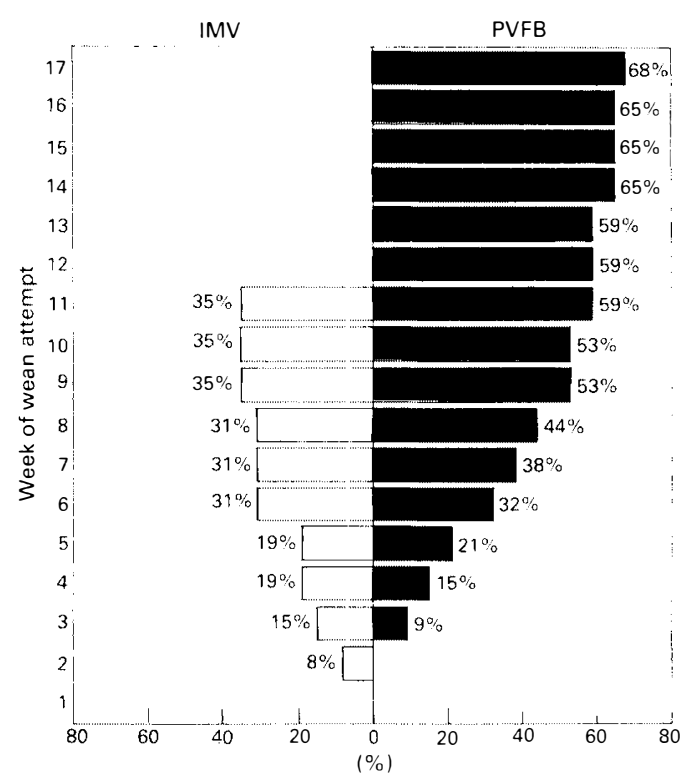

Figure 1 Cumulative percentage off ventilator, by method and week of attempt. methods with respect to the time elapsed until complete ventilator independence was achieved. It illustrates that IMV had a slightly greater success rate in the first 4 weeks, but that PVFB successes surpassed those of IMV after the fourth week. IMV failures also tended to occur early, and no IMV wean attempts extended beyond 11 weeks (either due to failure or discharge). On the other hand, PVFB wean attempts continued beyond 12 weeks with successes occurring through the 17 th week. When comparing only early attempts - those which were begun during the first month postinjury-IMV had a $32 \%$ success rate, compared with a success rate of $100 \%$ for PVFB $(p=0.01)$. However, it is important to note that there were only five cases in the latter group. Similarly, considering only cases for which weaning was begun 30 or more days postinjury, PVFB's success rate was still greater: $67 \%$ versus $43 \%$.

Additionally, there were 17 patients who underwent PVFB weaning attempts after having unsuccessful or uncompleted attempts with the IMV methodology. Twelve of the 17 were completely successful, one failed the PVFB method, and four were discharged to home still requiring part-time ventilator support.

\section{Discussion}

Several conclusions can be drawn from this study. First, despite the methodological limitations which prevented controlling for wean readiness criteria and type of mechanical ventilators used, the expectation that individuals with quadriplegia at the $\mathrm{C} 3$ and C4 levels can wean from ventilators, as reported elsewhere, ${ }^{13,15}$ is substantiated. Regardless of the weaning method utilized, this sample of 52 had an overall success rate of $83 \%$. In addition, another $12 \%$ attained at least partial ventilator independence. Second, even with this study's confounding factors, PVFB, with a $67.7 \%$ success rate compared with IMV's $34.6 \% \quad(p=0.02)$, seems a more effective means of weaning individuals with high-level quadriplegia. In evaluating the outcomes of the individuals who were discharged with their weaning still 
in progress, it is important to note that regardless of their outcomes PVFB would remain the most successful method. Indeed, even if all of those discharged still on IMV had successfully weaned during that trial, and all of those on PVFB went on to fail their wean attempts, the PVFB success rate still would be $68 \%$ compared with IMV's $54 \%$. Moreover, this study found PVFB to be more successful than IMV on several levels. In addition to its greater success rate overall, PVFB saw more success early postinjury, more success among individuals injured longer than a month, and a substantial number of successes among individuals who already had failed to wean using intermittent mandatory ventilation. Although this current study did find IMV to be faster initially, it also found that if IMV did not succeed early, it was less likely to succeed at all.

The superiority of the PVFB method was suggested over a decade ago by Sivak who felt T-piece weaning could be the method of choice both for patients with neuromuscular diseases and for those whose respiratory musculature was debilitated by prolonged mechanical ventilation: ${ }^{9}$ individuals with ventilator dependent quadriplegia meet both of these criteria. IMV, Sivak suggested, tends to allow patients to accommodate to less minute volume, retain carbon dioxide, and develop compensatory metabolic alkalosis, which blunts respiratory drive'. ${ }^{9}$ These factors may decrease the likelihood of IMV weaning success in the spinal cord injured patient.

Third, PVFB provides three frequently overlooked advantages not offered by other weaning methods. First, PVFB weaning can begin very early in the spinal cord injured individual's postinjury period, with durations as brief as 1 or 2 minutes, even when the patient's spontaneous vital capacity may be as low as approximately $100 \mathrm{cc}$. This allows the patient, staff, and family members to see and be encouraged by early, incremental progress. Second, PVFB has the social advantage of enabling individuals to utilize 'trach talking' with a one-way valve designed to permit exhaled air to pass over the vocal cords. Finally, as evidenced by the $12 \%$ of this study's participants who left the hospital only partially dependent on the mechanical ventilator, PVFB offers the functional benefit of at least short periods of ventilator independence. Whether ventilator free all day, for a few hours, or even just in the event of a machine failure, the individual who can survive without the ventilator has greater peace of mind, more discharge options, a less complicated home care scenario, and very likely a higher quality of life and countless choices not available to the individual who is truly ventilator dependent.

Several areas for additional research are suggested by this study. Further prospective evaluation of the two methods is clearly warranted, particularly to provide additional information about PVFB when used as a first weaning attempt, and to allow for controlling of such patient-dependent variables as wean readiness and such machinedependent variables as IMV airway resistance. In addition further examination of the complications associated with both methods is needed. Finally, research is needed to develop criteria for weaning readiness and to identify factors that are associated with successful weaning for both of the methods.

\section{Acknowledgement}

This research is supported, in part, by a grant from the National Institute for Disability and Rehabilitation Research (\#CFDA 84.133N)

\section{References}

1 Goldstone J, Moxham J (1991) Assisted ventilation: Weaning from mechanical ventilation. Thorax 46(1): $56-62$.

2 Yang KL, Tobin MJ (1991) A prospective study of indexes predicting the outcome of trials of weaning from mechanical ventilation. $N$ Engl J Med 324(21): 1445-1450.

3 Stone AM, Bone RC (1989) Successful weaning from mechanical ventilation. Postgrad Med 86(5): 315-319.

4 Knebel AR (1991) Weaning from mechanical ventilation: current controversies. Heart Lung 20(4): $321-331$. 
5 Coates NE, Weigelt JA (1991) Weaning from mechanical ventilation. Surg Clin North Am 71(4): 859-876.

6 Tobin MJ (1991) Weaning patients from mechanical ventilation. How to avoid difficulty. Postgrad Med 89(1): $171-178$.

7 Swinamer DL, Fedoruk LM, Jones RL, Chin WDN, Phang PT, Hamilton SM et al (1989) Energy expenditure associated with CPAP and T-piece spontaneous ventilatory trials. Changes following prolonged mechanical ventilation. Chest 96(4): 867-872.

8 Schacter EN, Tucker D, Beck GJ (1981) Does intermittent mandatory ventilation accelerate weaning? JAMA 246(11): 1210-1214.

9 Sivak ED (1980) Prolonged mechanical ventilation. An approach to weaning. Cleve Clin Q 47(2): 89-96.

10 Venus B, Smith RA, Mathru M (1987) National survey of methods and criteria used for weaning from mechanical ventilation. Crit Care Med 15: 530.

11 Feeley TW, Hedley-Whyte J (1975) Weaning from controlled ventilation and supplemental oxygen. $N$ Engl J Med 292(17): 903-905.

12 Ashutosh K (1983) Gradual versus abrupt weaning from respiratory support in acute respiratory failure and advanced chronic obstructive lung disease. South Med J 76(10): 1244-1248.

13 Carter RE (1987) Respiratory aspects of spinal cord injury management. Paraplegia 25(3): 262-266.

14 Bellany R, Pitts FW, Stauffer ES (1973) Respiratory complications in traumatic quadriplegia: analysis of 20 years`experience. J Neurosurg 39: 596-600.

15 Wicks AB, Menter RR (1986) Long-term outlook in quadriplegic patients with initial ventilator dependency. Chest 90: 406-410.

16 Lamid S, Ragalie GF, Welter K (1985) Respirator-dependent quadriplegics: problems during the weaning period. J Am Paraplegia Soc 8(2): 33-37.

17 Bach JR, Alba AS (1990) Noninvasive options for ventilatory support of the traumatic high level quadriplegia. Chest 98: 613-619.

18 Banner MJ, Blanch PB, Kirby RR (1993) Imposed work of breathing and methods of triggering a demand-flow, continuous airway pressure system. Crit Care Med 21(2): 183-190.

19 Sandercock PR (1989) The odds ratio: a useful tool in neurosciences. J Neurol Neurosurg Psychiatry 52: $817-820$. 